\begin{tabular}{|c|l|}
\hline Title & Sphingolipid synthesis is involved in autophagy in Saccharomyces cerevisiae \\
\hline Author(s) & Y amagata, Maki; Obara, Keisuke; Kinara, A kio \\
\hline Citation & $\begin{array}{l}\text { Biochemical and Biophysical Research Communications, 410(4), 786-791 } \\
\text { https://doi.org/10.1016j.bbrc.2011.06.061 }\end{array}$ \\
\hline Issue Date & 2011-07-15 \\
\hline Doc URL & http://hdl.handle.net/2115/46929 \\
\hline Type & article(author version) \\
\hline File Information & BBRC410-4_786-791.pdf \\
\hline
\end{tabular}

Instructions for use 


\section{Sphingolipid synthesis is involved in autophagy in Saccharomyces cerevisiae}

\section{Maki Yamagata, Keisuke Obara, Akio Kihara*}

Laboratory of Biochemistry, Faculty of Pharmaceutical Sciences, Hokkaido University,

Kita 12-jo, Nishi 6-choume, Kita-ku, Sapporo 060-0812, Japan

*Corresponding author

Address correspondence to:
Akio Kihara
Laboratory of Biochemistry
Faculty of Pharmaceutical Sciences, Hokkaido University
Kita 12-jo, Nishi 6-choume, Kita-ku, Sapporo 060-0812, Japan
Telephone: +81-11-706-3754
Fax: +81-11-706-4900
E-mail: kihara@pharm.hokudai.ac.jp 


\begin{abstract}
In eukaryotes, autophagy is a conserved protein degradation system that degrades cytoplasmic components by encompassing them with double-membrane structures, called autophagosomes, and delivering them to the lytic compartments of vacuoles/lysosomes. Certain Atg proteins are known to be involved in autophagy, yet the identity and function of lipid molecules involved remain largely unknown. We investigated the involvement of sphingolipids in autophagy using Saccharomyces cerevisiae. Inhibiting synthesis of the simplest complex sphingolipid, inositol phosphorylceramide (IPC), resulted in reduced autophagic activities. Similar results were obtained using myriocin, an inhibitor of the first step in sphingolipid synthesis. Our results indicate that sphingolipids, especially IPC, are required for autophagy. Inhibition of sphingolipid synthesis had no effect on formation of Atg12-Atg5 or Atg8-phosphatidylethanolamine conjugates, on maturation of vacuolar proteases, or on formation of the pre-autophagosomal structure (PAS). These results suggest that sphingolipids are not involved in the cellular signaling that leads to formation of the PAS, but may be involved in the process of autophagosome formation.
\end{abstract}

Keywords: Autophagy; Lipid; Sphingolipid 


\section{Abbreviations}

AbA, aureobasidin A; ALP, alkaline phosphatase; ApeI, aminopeptidase I; Cer, ceramide; CPY, carboxypeptidase Y; Cvt, cytoplasm to vacuole targeting; DHS, dihydrosphingosine; IPC, inositol phosphorylceramide; MIPC, mannosylinositol phosphorylceramide; $\mathrm{M}(\mathrm{IP})_{2} \mathrm{C}$, mannosyldiinositol phosphorylceramide; Myr, myriocin; PAS, pre-autophagosomal structure; PE, phosphatidylethanolamine; PHS, phytosphingosine; SC, synthetic complete; S(-NC), synthetic medium deprived nitrogen and carbon sources. 


\section{Introduction}

In autophagy, a bulk protein degradation system, cytoplasmic components are delivered to degradation compartments (vacuoles in yeast and lysosomes in mammals) and degraded. This system is conserved in eukaryotes. One well-defined physiological role of autophagy is to recycle amino acids, especially under starvation. A growing number of other physiological roles have been revealed by recent studies. Newly identified roles include removal of protein aggregates that might otherwise cause neuronal disorders such as Huntington's disease, extirpation of pathogenic bacteria, and antigen presentation [1].

Electron microscopic analyses of nutrient-deprived yeast have revealed details of the autophagic process [2,3]. A cup-shaped isolated membrane first appears in the cytosol, then extends, and finally encompasses a portion of cytoplasm, thereby generating a double-membraned structure called an autophagosome. The outer membrane of the autophagosome fuses with the vacuole membrane, releasing a single-membrane autophagic body, into the lumen of the vacuole. The hydrolases within the vacuole degrade the autophagic body and its contents to recycle their lipids and amino acids.

Over 30 autophagy-related proteins (Atg proteins) have been identified, 18 of which are essential for autophagosome formation [4-7]. Atg12 and Atg8 are ubiquitin-like molecules, and each covalently conjugates with a specific partner, Atg5 or phosphatidylethanolamine (PE), respectively [8-10]. Like the ubiquitin conjugation system, which utilizes three proteins (E1, E2, and E3) to attach ubiquitin to target 
proteins, Atg12 conjugation to Atg5 is mediated by the E1-like protein Atg7, and the E2-like protein Atg10. During Atg8-PE conjugation, the C-terminal Gly residue of Atg8 is cleaved by the protease Atg4, then the exposed C-terminus is conjugated to PE through successive actions of Atg7 and the E2-like Atg3 and the E3-like Atg12-Atg5 conjugate. The formation of the Atg8-PE conjugate is stimulated under nutrient-starvation, i.e. during induction of autophagy, and the conjugate is localized both on the extending isolation membranes and the autophagosome membranes $[11,12]$.

The Atg proteins functioning in autophagy have been analyzed extensively, yet with the exception of PE and phosphatidylinositol 3-phosphate [10,13], little is known about the lipid molecules that form autophagosomes. Sphingolipids are abundant lipid components of eukaryotic plasma membranes that function in a wide range of biological processes including proliferation, apoptosis, adhesion, skin barrier formation, and trafficking of immune cells [14]. These traits make sphingolipids strong candidates for study as possible autophagosomal lipids.

The backbone of sphingolipids, ceramide (Cer), is composed of a long-chain base attached to a fatty acid via an amide bond. The biosynthesis of sphingolipids begins with the condensation of serine with palmitoyl-CoA to form 3-ketodihydrosphingosine, which is then reduced to dihydrosphingosine (DHS) and acylated to dihydroceramide. Alternatively, a hydroxy group is attached to the 4-position of DHS, generating phytosphingosine (PHS). PHS and some DHS are then converted to Cers (dihydroceramide and phytoceramide) and further to inositol-containing complex sphingolipids, specifically inositol phosphorylceramide (IPC), mannosylinositol 
phosphorylceramide (MIPC), and mannosyldiinositol phosphorylceramide (M(IP) $\left.{ }_{2} \mathrm{C}\right)$ (Fig. 1) [14].

Cer and the sphngolipid metabolite sphingosine 1-phosphate reportedly induce autophagy in mammals, yet the causal molecular mechanisms are unclear. In yeast, a model organism in which autophagy has been extensively analyzed, certain autophagy process can be monitored or blocked by appropriate atg mutations. However, the functions of sphingolipids in yeast autophagy have rarely been studied. A single report described a slight increase ( $\sim 1.2$ fold $)$ in autophagic activity in the absence of $\mathrm{M}(\mathrm{IP})_{2} \mathrm{C}$, caused by a double deletion of the IPT1 gene, which encodes inositolphosphotransferase, and the $S K N 1$ gene, which is also involved in $\mathrm{M}(\mathrm{IP})_{2} \mathrm{C}$ synthesis through an unknown mechanism [15]. However, a single deletion mutantion of the IPT1 gene had no effect [15].

To address this nearly total lack of information regarding autophagosomal lipid biology in yeast, we investigated the involvement of sphingolipids using mutants of sphingolipid biosynthesis genes and inhibitors of sphingolipid synthesis. We found that blocking 3-ketodihydrosphingosine synthesis, the first step of sphingolipid synthesis, or IPC synthesis results in reduced autophagic activity, whereas inhibition of MIPC or $\mathrm{M}(\mathrm{IP})_{2} \mathrm{C}$ synthesis has no effect. This study affirms that sphingolipid synthesis is indeed required for normal progression of autophagy. 


\section{Materials and methods}

\subsection{Yeast strain and media}

The Saccharomyces cerevisiae strains used in this study are listed in Table I. Cells were grown in either YPD medium (1\% yeast extract, $2 \%$ peptone, and 2\% D-glucose) or synthetic complete (SC) medium $(0.67 \%$ yeast nitrogen base without amino acids (Sigma, St. Louis, MO), 2\% D-glucose, $0.5 \%$ casamino acid, $20 \mathrm{mg} / 1$ tryptophan, 20 $\mathrm{mg} / \mathrm{l}$ adenine, and $20 \mathrm{mg} / \mathrm{l}$ uracil) as a rich medium. Nitrogen- and carbon-deprived synthetic medium $(\mathrm{S}(-\mathrm{NC}) ; 0.17 \%$ yeast nitrogen base without amino acids and ammonium sulfate (Sigma)) was used to induce starvation. Aureobasidin A (AbA) and myriocin (Myr) were purchased from Takara Bio (Sigma, Japan) and Sigma, respectively.

Gene disruption of the CSG1, CSH1, or IPT1 gene was performed as follows. S.

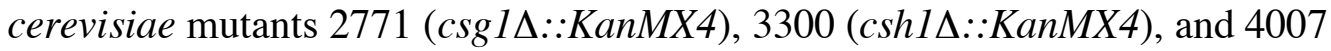
(ipt1 $1:: K a n M X 4)$ were obtained from Open Biosystems (Huntsville, AL). Gene fragments $\operatorname{csg} 1 \Delta:: K a n M X 4, \operatorname{csh} 1 \Delta:: K a n M X 4$, and ipt $1 \Delta:: K a n M X 4$, which each encompass both 5'- and 3'-untranslated regions with more than $200 \mathrm{bp}$, were amplified from genomic DNA prepared from their respective mutant cells, and were used for homologous recombination to disrupt each gene under desired genetic backgrounds.

\subsection{Assays for autophagy}

Detection of autophagy was performed by microscopic observation of autophagic bodies accumulated in vacuoles, or by alkaline phosphatase (ALP) assays [2,16]. For 
observation of autophagic bodies, the vacuolar protease-deficient yeast strains BJ2168 and BJ3505 and their derivatives were used. Cells grown in rich medium to log-phase $\left(\mathrm{OD}_{600} \geq 1\right)$ were transferred to $\mathrm{S}(-\mathrm{NC})$ medium. After incubation for $5 \mathrm{hr}$ at $30^{\circ} \mathrm{C}$, cells were observed using a phase-contrast microscope IX-81 (Olympus, Tokyo, Japan). A minimum of 400 cells for each mutant strain were examined, and cells accumulating autophagic bodies were counted. ALP assays were performed as described previously [17], using yeast strains derived from KVY55, which expresses a mutant ALP lacking a signal sequence.

\subsection{Immunoblotting}

Total cell lysates were prepared by the alkaline/trichloroacetic acid method as described previously [17]. Lysates equivalent to $0.3 \mathrm{OD}_{600}$ were subjected to SDS-PAGE. For detection of Atg8, $6 \mathrm{M}$ urea was included in the separation gel to separate Atg8 and PE-conjugated Atg8 [9]. Proteins were then transferred to Immobilon ${ }^{\mathrm{TM}}$ polyvinylidene difluoride membrane (Millipore, Billerica, MA). The membrane was then incubated with an anti-Atg12 antibody (1:2000 dilution [13]), anti-Atg8 antibody (1:2500 dilution [9], anti-Ape1 antibody (1:5000 dilution [18]), anti-Pep4 antibody (1:5000 dilution), anti-CPY antibody $(0.25 \mu \mathrm{g} / \mathrm{ml}$; Molecular Probes, Eugene, OR), or anti-Pgk1 antibody $(0.25 \mu \mathrm{g} / \mathrm{ml}$; Molecular Probes) for $1 \mathrm{hr}$, then with HRP-conjugated anti-rabbit or anti-mouse IgG $\mathrm{F}(\mathrm{ab})_{2}$ fragment (each from GE Healthcare Bio-Sciences, Piscataway, $\mathrm{NJ}$ and diluted 1:10,000) for $1 \mathrm{hr}$. Labeling was detected using $\mathrm{ECL}^{\mathrm{TM}}$ Reagents or an ECL Plus System for Western Blotting Detection (GE Healthcare Bio-Sciences) using 
X-ray films. The Pep4 signal was enhanced using Can Get Signal Immunoreaction Enhancer Solution (TOYOBO, Osaka, Japan).

\subsection{Fluorescence microscopy}

Yeast cells (strain ORY1700) chromosomally expressing Atg17-GFP underwent fluorescence microscopic analyses. Cells grown in rich medium to log-phase were transferred to $\mathrm{S}(-\mathrm{NC})$ medium. After a $2.5 \mathrm{hr}$ incubation, the cells were analyzed by fluorescence microscopy using an HS All-in-one Fluorescence Microscope BZ-9000 (Keyence, Osaka, Japan). 


\section{Results}

3.1. Sphingolipid synthesis is required for normal progression of autophagy

To examine the involvement of sphingolipids in autophagy, we used myriocin (Myr), an inhibitor for serine palmitoyltransferase, which catalyzes the first step of sphingolipid synthesis, and aureobasidin A (AbA), an inhibitor for IPC synthase. Autophagy induction in yeast is often investigated under nitrogen-starved conditions. However, we found that yeast cells undergo rapid cell death in AbA combined with nitrogen-starvation medium (our unpublished data). Instead, we used nitrogen- and carbon-deprived medium S(-NC), which did not induce cell death even in the presence of AbA.

We first examined the accumulation of autophagic bodies in vacuoles of yeast cells that lack most vacuolar protease activities and so are unable to degrade autophagic bodies. Under nitrogen- and carbon-starved conditions, wild type cells with mock treatment accumulated autophagic bodies in vacuoles. The negative control atg $14 \Delta$ cells, which cannot form autophagosomes [19], formed no autophagic bodies under similar conditions. When AbA was added to wild type cells, the number of cells accumulating autophagic bodies was reduced (Fig. 2A and B). In addition, both the number and size of autophagic bodies present were decreased in the presence of AbA (Fig. 2A). Similar results were obtained using Myr, and the number of cells accumulating autophagic bodies was reduced (Fig. 2B).

We next examined autophagic activities by measuring the activity of an alkaline phosphatase (ALP) Pho8 mutant protein (Pho8 460$)$ [16]. Wild type Pho8 protein is 
synthesized in the ER, transported to the vacuole, and maturated by processing. Since Pho8 860 lacks a targeting sequence for the vacuole, it is mislocalized to the cytosol. Pho8 860 must enter the vacuole by autophagy, so measureing Pho8 activity represents autophagic activity. Mock-treated wild type cells but not atg $14 \Delta$ cells exhibited increased ALP activity when cells were shifted from growth conditions to starvation (Fig. 2C). Treatment of wild type cells with AbA significantly reduced the ALP activity, although not completely. Myr also caused a reduction in ALP activity under starvation (Fig. 2C). These results indicate that sphingolipid synthesis is required for normal progression of autophagy.

\section{2. $\mathrm{MIPC}$ and $\mathrm{M}(\mathrm{IP})_{2} \mathrm{C}$ are not required for autophagy}

AbA treatment inhibits IPC synthesis. Since IPC is a precursor for MIPC and M(IP) $)_{2} \mathrm{C}$, treatment with $\mathrm{AbA}$ also causes a reduction in their levels. We next examined the involvement of MIPC or M(IP $)_{2} \mathrm{C}$ in autophagy. MIPC synthesis is catalyzed by two redundant enzymes, Csg1 and Csh1. M(IP) $)_{2} \mathrm{C}$ synthesis is catalyzed by Ipt1, and its deletion reportedly had no effect on autophagy [15], which we confirmed in further detail here. Both $\operatorname{csg} 1 \Delta \operatorname{csh} 1 \Delta$ double mutant cells and ipt $1 \Delta$ cells are viable, in contrast to mutants of the essential AUR1 gene, which encodes IPC synthase. The $\operatorname{csg} 1 \Delta \operatorname{csh} 1 \Delta$ cells and ipt $1 \Delta$ cells were grown in $\mathrm{S}(-\mathrm{NC})$ medium and subjected to microscopic analysis. Both cells accumulated autophagic bodies similarly to wild type cells (Fig. 3A and B). Moreover, both the number and size of the autophagic bodies were indistinguishable from those in the wild type cells. An ALP assay also demonstrated 
that the $\operatorname{csg} 1 \Delta \operatorname{csh} 1 \Delta$ cells or ipt $1 \Delta$ cells normally initiate autophagy in response to starvation (Fig. 3C). Thus, MIPC and M(IP) ${ }_{2} \mathrm{C}$ are not necessary for autophagy.

3.3. Sphingolipids are not required for formation of Atg12-Atg5, Atg8-PE, or the pre-autophagosomal structure

We next investigated which processes of autophagy involve sphingolipids. Two essential components of autophagy, Atg12 and Atg8, are ubiquitin-like proteins that form covalently linked conjugates with their specific partners, Atg5 and PE, respectively. We investigated the effects of sphingolipid inhibitors on Atg12-Atg5 and Atg8-PE formation. Treatment of wild type cells or atg14 cells with Myr, AbA, or a combination thereof had no effect on Atg12-Atg5 or Atg8-PE formation, regardless of the culture conditions, indicating that sphingolipids are not required for Atg12-Atg5 or Atg8-PE formation (Fig. 4A).

Some vacuolar proteins, such as aminopeptidase I (ApeI), are targeted to the vacuole through a mechanism related to autophagy, called Cvt (cytoplasm to vacuole targeting) [20]. In this pathway, ApeI is encompassed with a double membrane structure, the Cvt vesicle, then delivered to the vacuole via fusion. In contrast to autophagy, the Cvt pathway is active under nutrient-rich conditions [21,22]. The proteins required for the Cvt pathway and autophagy largely overlap [23], so we examined the involvement of sphingolipids in the Cvt pathway. ApeI is synthesized in the cytosol as a premature form and is converted to its mature form after entering the vacuole via the Cvt pathway, which is still active under nutrient-rich conditions. Mature protein was present in cells 
grown in rich medium, and the levels of mature protein increased under starvation in

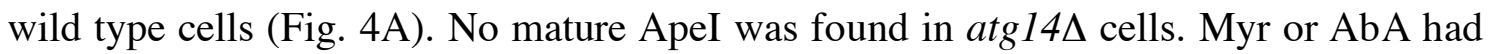
no effect on the maturation of ApeI in wild type cells in nutrient-rich medium. However, under starvation, treatment of cells with Myr or AbA caused a slight increase in the premature form of ApeI. These results suggest that sphingolipids are not essential for the Cvt pathway but they might have some small role.

Pep4 is a vacuolar protease important in the hydrolytic activities of vacuoles. This protease is involved in the maturation of most vacuolar proteins, including itself and carboxypeptidase Y (CPY), through enzymatic processing. Both Pep4 and CPY are synthesized in the ER and delivered to vacuoles by vesicular transport. Levels of mature Pep4 and mature CPY were unchanged following treatment with Myr or AbA (Fig. 4A). This indicates that Pep4 activity was unaffected by the inhibition of sphingolipid synthesis. These results further suggest that the decreased autophagic activity caused by AbA or Myr was not due to insufficient vacuolar protease activities but rather to an effect on the autophagic process itself.

During the early step of autophagosome formation, some Atg proteins are clustered at the pre-autophagosomal structure (PAS) adjacent to the vacuole. Atg17 plays a pivotal role in PAS formation [24,25]. Localization of Atg17 to the PAS is stimulated during autophagy induction, so monitoring Atg17 is useful in evaluating PAS formation. To investigate the effect of sphingolipid synthesis on PAS formation, we observed the localization of Atg17-GFP in the presence of AbA or Myr. In mock-treated wild type cells under starvation, Atg17-GFP was observable in dotted 
structures, the typical pattern of PAS (Fig. 4B). This pattern was also apparent following treatment with AbA or Myr (Fig. 4B). Thus, sphingolipid synthesis is not required for autophagy-induced PAS formation. 


\section{Discussion}

Sphingolipids have a variety of cellular functions other than just being a constituent of biological membranes. However, until now, their involvement in autophagy has not been examined. In the presented study using budding yeast, we demonstrated that sphingolipids are necessary for the normal progression of autophagy.

Inhibiting the synthesis of the simplest complex sphingolipid (IPC), using the inhibitor AbA, caused a significant reduction in autophagic activity (Fig. 2). However, deletion of the genes responsible for MIPC or M(IP $)_{2} \mathrm{C}$ synthesis had no effect on the induction of autophagy (Fig. 3). Thus, IPC synthesis itself may be involved in autophagy. Logically, treatment with AbA causes not only a reduction in IPC levels but also an increase in its substrate Cer. Therefore, it is possible that an accumulation of Cer might cause inhibition of autophagy. However, inhibiting the first step of sphingolipid synthesis using the inhibitor Myr also resulted in an inhibition of autophagy (Fig. 2B and C), suggesting that reduced IPC but not accumulation of Cer is the cause of the autophagy defect.

Increased Cer reportedly causes autophagy in mammalian cells [26], although it is still unclear whether Cer or its metabolites, such as complex sphingolipids, are involved. We demonstrated here that in yeast the complex sphingolipid IPC is important for autophagy. Although IPC, in which phosphoinositol is attached to Cer, does not exist in mammals, mammals do contain a similar complex sphingolipid, sphingomyelin, in which phosphocholine is attached to Cer. Future stiduies into the involvement of sphingomyelin in autophagy in mammals will be required to elucidate the evolutional 
conservation of autophagy-inducing mechanisms.

In the experimental conditions here, wild type cells grown to log-phase in nutrient-rich medium were incubated with AbA or Myr for a relatively short period (30 min), then were shifted to $\mathrm{S}(-\mathrm{NC})$ medium containing $\mathrm{AbA}$ or $\mathrm{Myr}$, and further incubated. Under starvation with S(-NC) medium, the cell cycle is immediately stopped, and dilution of pre-existing sphingolipids, including IPC, by cell division should be minimal. We speculate that newly synthesized IPC but not stored cellular sphingolipids are important for autophagy.

Cells treated with AbA or Myr still formed Atg8-PE conjugates in response to starvation (Fig. 4A). In addition, Atg17, the core protein for clustering of Atg proteins, localized normally in the PAS in the presence of AbA or Myr (Fig. 4B). These results suggest that sphingolipids may not be involved in the signaling process that links starvation conditions to the cellular response of autophagy. On the other hand, $\mathrm{AbA}$ and Myr each caused a reduction in the number and size of autophagic bodies (Fig. 2A and B), suggesting that sphingolipid synthesis is directly involved in the autophagosome formation. Interestingly, AbA and Myr each had only a slight effect on the maturation of ApeI (Fig. 4A). The Cvt pathway and autophagy are highly analogous in their mechanism, and most proteins involved in their induction are shared [23]. The most striking difference between a Cvt vesicle and an autophagosome is in size. An average autophagosome is $500 \mathrm{~nm}$ in diameter, a Cvt vesicle $150 \mathrm{~nm}$ [20]. Although ApeI is transported to the vacuole via the Cvt pathway under growth conditions, autophagosomes also deliver ApeI to the vacuole during autophagy [20]. 
Autophagosomes with a decreased size caused by AbA or Myr treatment may still possess the capacity to deliver ApeI to the vacuole.

The lipid compositions of autophagosomes have not been determined so far. Our results imply that sphingolipids are autophagosome membrane components. Further studies are needed to determine whether sphingolipids function in supplying lipid molecules for autophagosome formation, in determination of autophagosome size, or in recruitment of proteins essential for autophagy progression. 


\section{Acknowledgments}

We thank Dr. Y. Ohsumi (Frontier Research Center, Tokyo Institute of Technology) for providing yeast strains and anti-Atg12, anti-Atg8, anti-ApeI and anti-Pep4 antibodies. We are grateful to Dr. E. A. Sweeney for scientific editing of the manuscript. This work was supported by a Grant-in-aid for Young Scientists (B) (21770129) from the Ministry of Education, Culture, Sports, Science and Technology of Japan. 


\section{References}

[1] N. Mizushima, Autophagy: process and function, Genes Dev. 21 (2007) 2861-2873.

[2] K. Takeshige, M. Baba, S. Tsuboi, et al., Autophagy in yeast demonstrated with proteinase-deficient mutants and conditions for its induction, J. Cell Biol. 119 (1992) 301-311.

[3] M. Baba, K. Takeshige, N. Baba, et al., Ultrastructural analysis of the autophagic process in yeast: detection of autophagosomes and their characterization, J. Cell Biol. 124 (1994) 903-913.

[4] M. Tsukada, Y. Ohsumi, Isolation and characterization of autophagy-defective mutants of Saccharomyces cerevisiae, FEBS Lett. 333 (1993) 169-174.

[5] M. Thumm, R. Egner, B. Koch, et al., Isolation of autophagocytosis mutants of Saccharomyces cerevisiae, FEBS Lett. 349 (1994) 275-280.

[6] D.J. Klionsky, J.M. Cregg, W.A. Dunn, Jr., et al., A unified nomenclature for yeast autophagy-related genes, Dev. Cell 5 (2003) 539-545.

[7] H. Nakatogawa, K. Suzuki, Y. Kamada, et al., Dynamics and diversity in autophagy mechanisms: lessons from yeast, Nat. Rev. Mol. Cell Biol. 10 (2009) 458-467.

[8] N. Mizushima, T. Noda, T. Yoshimori, et al., A protein conjugation system essential for autophagy, Nature 395 (1998) 395-398.

[9] T. Kirisako, Y. Ichimura, H. Okada, et al., The reversible modification regulates the membrane-binding state of Apg8/Aut7 essential for autophagy and the 
cytoplasm to vacuole targeting pathway, J. Cell Biol. 151 (2000) 263-276.

[10] Y. Ichimura, T. Kirisako, T. Takao, et al., A ubiquitin-like system mediates protein lipidation, Nature 408 (2000) 488-492.

[11] T. Kirisako, M. Baba, N. Ishihara, et al., Formation process of autophagosome is traced with Apg8/Aut7p in yeast, J. Cell Biol. 147 (1999) 435-446.

[12] Y. Kabeya, N. Mizushima, T. Ueno, et al., LC3, a mammalian homologue of yeast Apg8p, is localized in autophagosome membranes after processing, EMBO J. 19 (2000) 5720-5728.

[13] K. Obara, T. Noda, K. Niimi, et al., Transport of phosphatidylinositol 3-phosphate into the vacuole via autophagic membranes in Saccharomyces cerevisiae, Genes Cells 13 (2008) 537-547.

[14] A. Kihara, S. Mitsutake, Y. Mizutani, et al., Metabolism and biological functions of two phosphorylated sphingolipids, sphingosine 1-phosphate and ceramide 1-phosphate, Prog. Lipid Res. 46 (2007) 126-144.

[15] K. Thevissen, W.L. Yen, D. Carmona-Gutierrez, et al., Skn1 and Ipt1 negatively regulate autophagy in Saccharomyces cerevisiae, FEMS Microbiol. Lett. 303 (2010) 163-168.

[16] T. Noda, A. Matsuura, Y. Wada, et al., Novel system for monitoring autophagy in the yeast Saccharomyces cerevisiae, Biochem. Biophys. Res. Commun. 210 (1995) 126-132.

[17] K. Obara, T. Sekito, Y. Ohsumi, Assortment of phosphatidylinositol 3-kinase complexes-Atg14p directs association of complex I to the pre-autophagosomal 
structure in Saccharomyces cerevisiae, Mol. Biol. Cell 17 (2006) 1527-1539.

[18] K. Suzuki, Y. Kamada, Y. Ohsumi, Studies of cargo delivery to the vacuole mediated by autophagosomes in Saccharomyces cerevisiae, Dev. Cell 3 (2002) 815-824.

[19] S. Kametaka, T. Okano, M. Ohsumi, et al., Apg14p and Apg6/Vps30p form a protein complex essential for autophagy in the yeast, Saccharomyces cerevisiae, J. Biol. Chem. 273 (1998) 22284-22291.

[20] M. Baba, M. Osumi, S.V. Scott, et al., Two distinct pathways for targeting proteins from the cytoplasm to the vacuole/lysosome, J. Cell Biol. 139 (1997) $1687-1695$.

[21] T. Yoshihisa, Y. Anraku, A novel pathway of import of $\alpha$-mannosidase, a marker enzyme of vacuolar membrane, in Saccharomyces cerevisiae, J. Biol. Chem. 265 (1990) 22418-22425.

[22] D.J. Klionsky, R. Cueva, D.S. Yaver, Aminopeptidase I of Saccharomyces cerevisiae is localized to the vacuole independent of the secretory pathway, J. Cell Biol. 119 (1992) 287-299.

[23] S.V. Scott, A. Hefner-Gravink, K.A. Morano, et al., Cytoplasm-to-vacuole targeting and autophagy employ the same machinery to deliver proteins to the yeast vacuole, Proc. Natl. Acad. Sci. U S A 93 (1996) 12304-12308.

[24] K. Suzuki, T. Kirisako, Y. Kamada, et al., The pre-autophagosomal structure organized by concerted functions of APG genes is essential for autophagosome formation, EMBO J. 20 (2001) 5971-5981. 
[25] K. Suzuki, Y. Kubota, T. Sekito, et al., Hierarchy of Atg proteins in pre-autophagosomal structure organization, Genes Cells 12 (2007) 209-218.

[26] F. Scarlatti, C. Bauvy, A. Ventruti, et al., Ceramide-mediated macroautophagy involves inhibition of protein kinase B and up-regulation of beclin 1, J. Biol. Chem. 279 (2004) 18384-18391.

[27] J.S. Robinson, D.J. Klionsky, L.M. Banta, et al., Protein sorting in Saccharomyces cerevisiae: isolation of mutants defective in the delivery and processing of multiple vacuolar hydrolases, Mol. Cell Biol. 8 (1988) 4936-4948. 


\section{Figure legends}

Fig. 1. The sphingolipid synthesis pathway of yeast. The pathway and responsible enzymes for yeast sphingolipid synthesis are shown. Processes inhibited by Myr and $\mathrm{AbA}$ are indicated. In Cer synthesis, a very long-chain fatty acid is conjugated to DHS or PHS via an amide bond.

Fig. 2. Sphingolipid synthesis is required for normal progression of autophagy. (A) BJ2168 (wild type) and MYY46 (atg14D) cells were grown in YPD medium to logarithmic growth phase. Cells were incubated with or without AbA for $30 \mathrm{~min}$. Starvation was induced by transferring cells to S(-NC) medium with or without AbA. After $5 \mathrm{hr}$, cells were observed under a phase contrast microscope. Photomicrographs of cells indicate the presence of autophagic bodies in mock-treated wild type cells. Bar indicates $5 \mu \mathrm{m}$. (B) BJ3505 (wild type) cells were cultured and treated as in (A) with or without AbA or Myr, as indicated. The frequency of cells with autophagic bodies was calculated by counting a minimum of 400 cells in each experiment, and determining the percentage. Values are the mean \pm S.D. from three independent experiments. Statistically significant differences are indicated (*, p<0.05; t-test). (C) KVY55 (wild

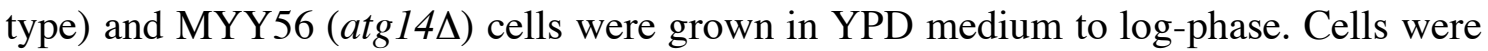
incubated with no inhibitor, AbA, or Myr for $30 \mathrm{~min}$ (R). Starvation was induced by transferring cells to S(-NC) medium with or without the inhibitor and incubating for 4 hr (-NC). Cell lysates were prepared and subjected to ALP assays. Error bars indicate standard deviations $(\mathrm{n}=3)$. Asterisks indicate significant differences $(* *, p<0.01)$. 
Fig. 3. MIPC synthesis and $\mathrm{M}(\mathrm{IP})_{2} \mathrm{C}$ synthesis are dispensable for autophagy. (A) BJ2168 (wild type) and MYY100 ( $\operatorname{csg} 1 \Delta \operatorname{csh} 1 \Delta)$ cells at logarithmic growth phase were transferred to $\mathrm{S}(-\mathrm{NC})$ medium. After $5 \mathrm{hr}$, cells were observed under a phase contrast microscope. Photomicrographs of cells indicate the presence of autophagic bodies in wild type and in $\operatorname{csg} 1 \Delta \operatorname{csh} 1 \Delta$ cells. Bar indicates $5 \mu \mathrm{m}$. (B) BJ3505 (wild type),

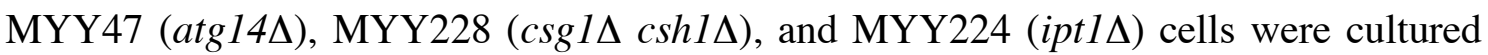
and treated as in (A). The frequency of cells with autophagic bodies was calculated by counting a minimum of 400 cells and determining the percentage. Error bars indicate standard deviations $(\mathrm{n}=3)$. (C) Autophagic activity was measured by an ALP assay


MYY226 (ipt1D) cells. Cells grown in YPD medium to log-phase were assayed (R) or were transferred to S(-NC) medium, cultured for $4 \mathrm{hr}$, and then assayed (-NC). Error bars indicate standard deviations $(\mathrm{n}=6)$. Asterisks indicate significant differences $(* *, \mathrm{p}$ $<0.01)$.

Fig. 4. Sphingolipid synthesis is not involved in the early signaling processes of autophagy or in Cvt. (A) KVY55 (wild type) and MYY56 (atg144) cells were grown in YPD medium to log-phase. Cells were incubated with no inhibitor, AbA, or Myr for 30 min (R). Starvation was induced by transferring cells to S(-NC) medium with or without the inhibitor for $4 \mathrm{hr}(-\mathrm{NC})$. Cell lysates were prepared and subjected to immunoblot analysis with an anti-Atg12 antibody, anti-Atg8 antibody, anti-ApeI antibody, anti-Pep4 
antibody, anti-CPY antibody, or, to demonstrate uniform protein loading, an anti-Pgk1 antibody. pApeI, premature API; mApeI, mature API. (B) ORY1700 cells, which express Atg17-GFP from a chromosome, were treated with methanol (mock), AbA, or Myr, for $2.5 \mathrm{hr}$ in $\mathrm{S}(-\mathrm{NC})$ medium, then observed under a fluorescence microscope. Atg17-GFP is apparent as dotted structures, the typical pattern of PAS. Bar indicates 5 $\mu \mathrm{m}$. 




Yamagata et al., Fig. 1 

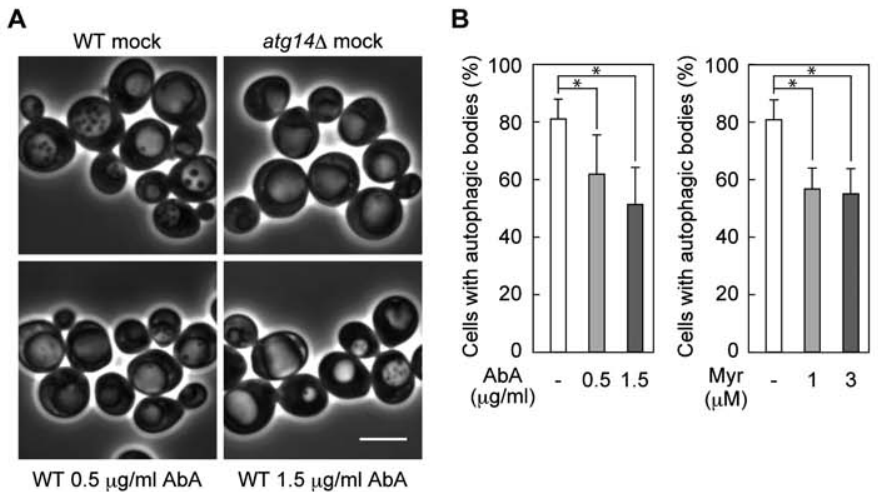

C
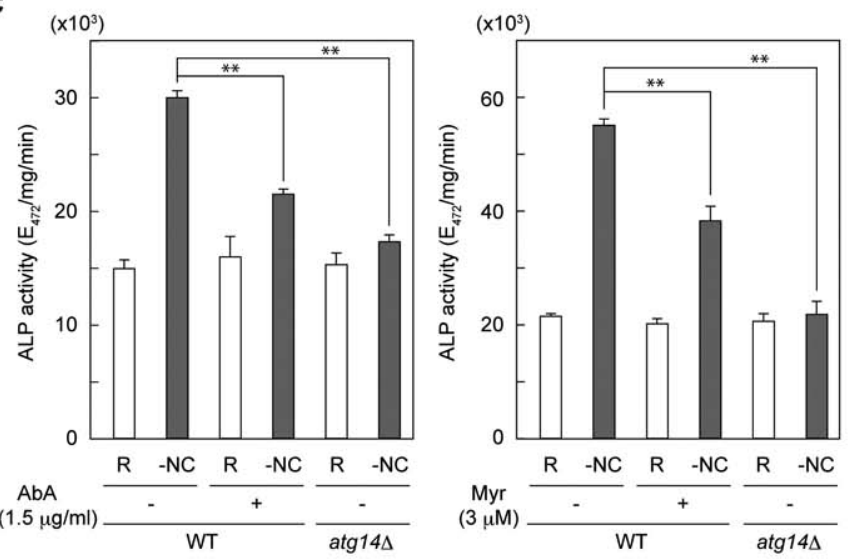

Yamagata et al., Fig. 2 


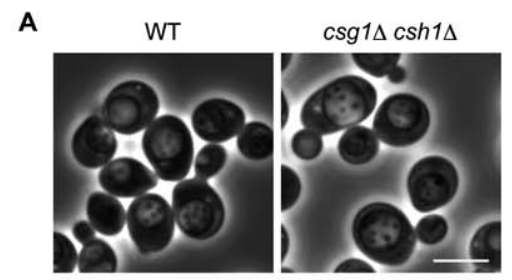

B

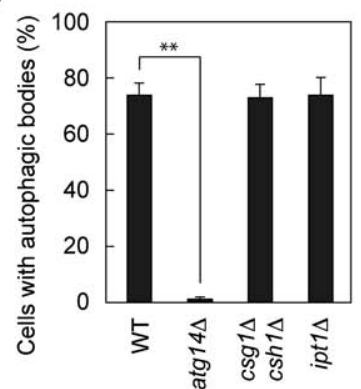

C

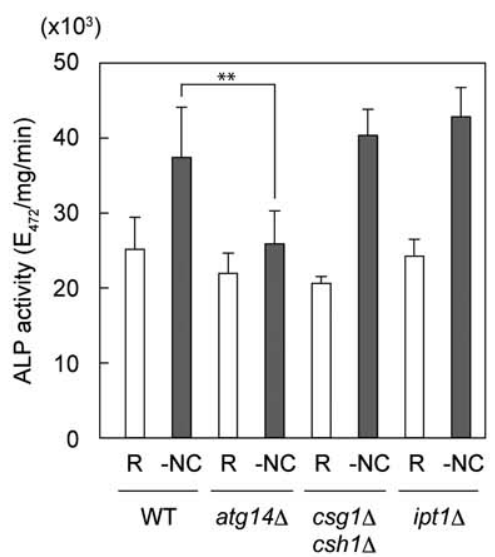

Yamagata et al., Fig. 3 


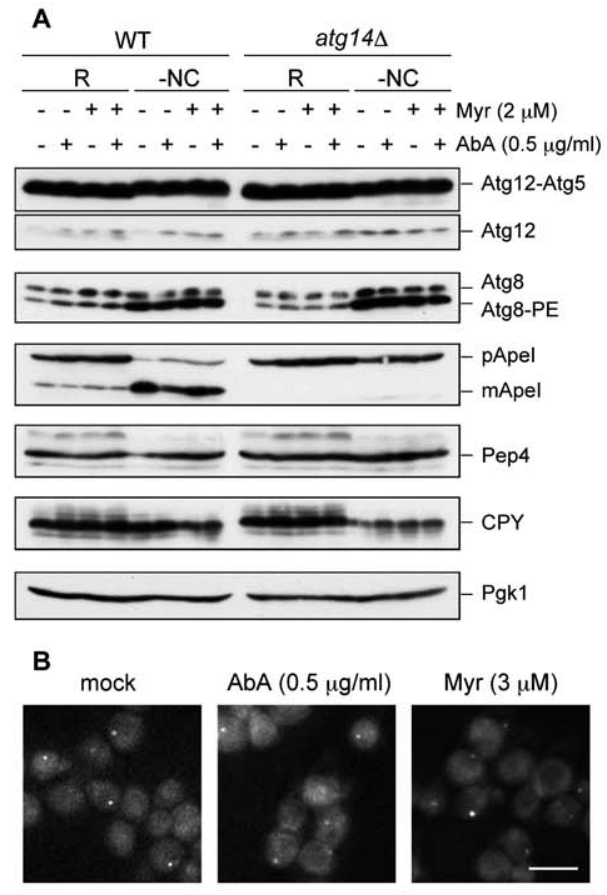

Yamagata et al., Fig. 4 